\title{
How Much Time are Physicians and Nurses Spending Together at the Patient Bedside?
}

\author{
Adam X Sang, MD*; Rebecca L Tisdale, MD²; Derek Nielsen, AA, BMET³; Silvia Loica-Mersa, MD²;

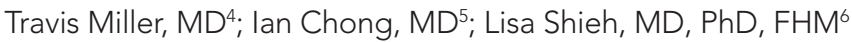

\begin{abstract}
${ }^{1}$ Department of Surgery, Stanford University School of Medicine, Stanford, California; ${ }^{2}$ Department of Medicine, Stanford University School of Medicine, Stanford, California; ${ }^{3}$ Clinical Technology, Stanford Hospital and Clinics, Stanford, California; ${ }^{4}$ Division of Plastic Surgery, Stanford University School of Medicine, Stanford, California; ${ }^{5}$ Department of Emergency Medicine, Stanford University School of Medicine, Stanford, California; ${ }^{6}$ Medical Director for Quality, Department of Medicine, Stanford University School of Medicine, Stanford, California.
\end{abstract}

BACKGROUND: Bedside rounding involving both nurses and physicians has numerous benefits for patients and staff. However, precise quantitative data on the current extent of physician-nurse (MD-RN) overlap at the patient bedside are lacking.

OBJECTIVE: This study aimed to examine the frequency of nurse and physician overlap at the patient beside and what factors affect this frequency.

DESIGN: This is a prospective, observational study of time-motion data generated from wearable radio frequency identification (RFID)-based locator technology.

SETTING: Single-institution academic hospital.

MEASUREMENTS: The length of physician rounds, frequency of rounds that include nurses simultaneously at the bedside, and length of MD-RN overlap were measured and analyzed by ward, day of week, and distance between patient room and nursing station.

RESULTS: A total of $739 \mathrm{MD}$ rounding events were captured over 90 consecutive days. Of these events, 267 took place in single-bed patient rooms. The frequency of MD-RN overlap was $30.0 \%$, and there was no statistical difference between the three wards studied. Overall, the average length of all MD rounds was $7.31 \pm 0.58$ minutes, but rounding involving a bedside nurse lasted longer than rounds with MDs alone (9.56 vs 5.68 minutes, $P<.05$ ). There was no difference in either the length of rounds or the frequency of MD-RN overlap between weekdays and weekends. Finally, patient rooms located farther away from the nursing station had a lower likelihood of MD-RN overlap (Pearson's $r=-0.67, P<.05$ ).

CONCLUSION: RFID-based technology provides precise, automated, and high-throughput time-motion data to capture nurse and physician activity. At our institution, 30.0\% of rounds involve a bedside nurse, highlighting a potential barrier to bedside interdisciplinary rounding. Journal of Hospital Medicine 2019;14:468-473. Published online first May 10, 2019. (C) 2019 Society of Hospital Medicine

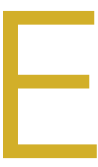

ffective communication between physicians and nurses is an essential element of any healthcare system. Numerous studies have highlighted the benefits of high quality physician-nurse (MD-RN) communication, including improved patient outcomes, ${ }^{1}$ higher patient satisfaction, ${ }^{2}$ and better nurse job satisfaction and retention rates. ${ }^{3-5}$ Having physicians and nurses round together (bedside interdisciplinary rounding) has been shown to improve the perception of teamwork, ${ }^{6,7}$ reduce the number of pages for the physician team, ${ }^{6,8}$ better involve the patients in developing the plan of care, ${ }^{8}$ and even decrease the length and cost of stay. ${ }^{9}$

Being physically in the same space at the same time is the first and nonnegotiable requirement of bedside interdisciplinary rounding. However, precise and objective data re-

*Corresponding Author: Adam X Sang, MD; E-mail: adam.sang@stanford.edu.

Received: November 10, 2018; Revised: March 1, 2019;

Accepted: March 13, 2019

๑) 2019 Society of Hospital Medicine DOI 10.12788/jhm.3204 garding the extent to which physicians and nurses overlap at the patient bedside are lacking. Studies that examine the face-to-face component of MD-RN communication have generally relied on either qualitative methods, such as focus groups and surveys, ${ }^{10,11}$ or quantitative methods that are subjective, such as validated scales. ${ }^{12}$ In addition, the few studies that report quantitative data usually rely on manual observation methods that can be affected by various forms of observer bias. ${ }^{10,13,14}$ There is also a paucity of data on how bedside overlap changes over the work week or as a function of room location.

Recently, real-time locator systems using radio frequency identification (RFID) have allowed measurement of staff and equipment movement in a precise and quantitative manner. ${ }^{9,15}$ Although there have been previous studies using RFID locators to create time-motion maps of various hospital staff, no study has used RFID to measure and analyze the workflow of both physicians and nurses simultaneously.16-18 The purpose of our investigation was to utilize our hospital-wide RFID staff locator technology to accurately and quantitatively assess physician and nurse rounding habits. Understanding the current 


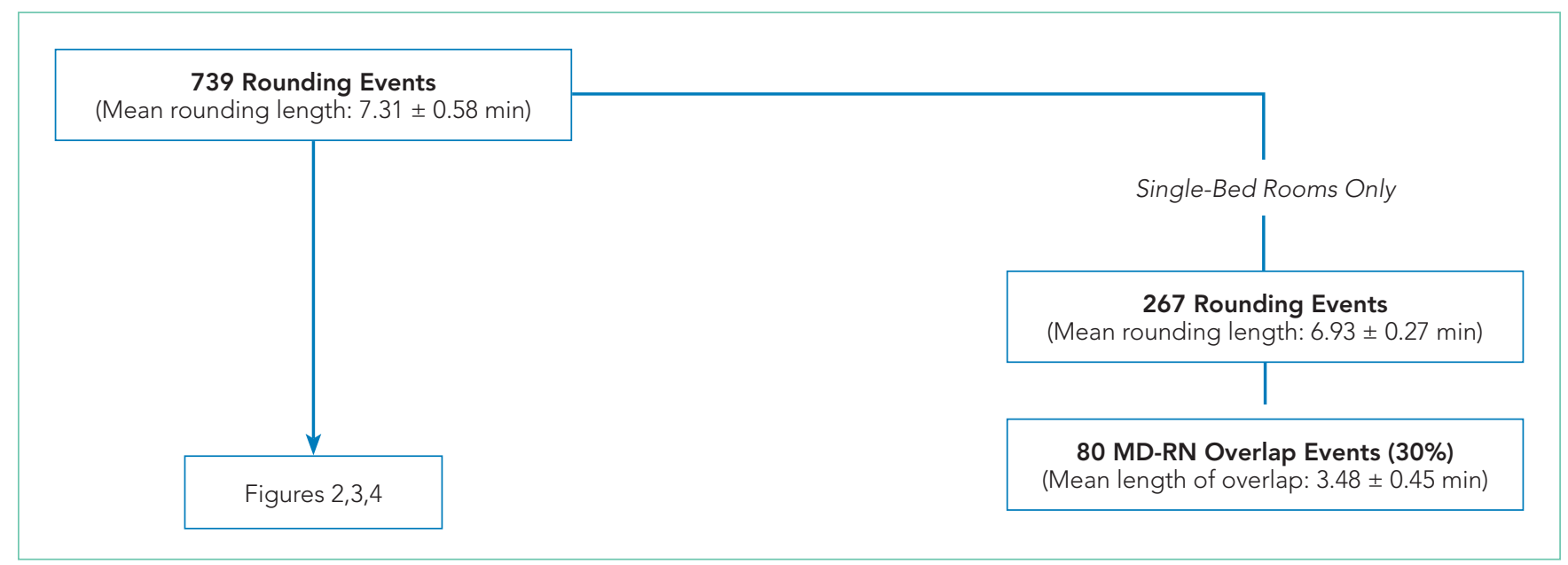

FIG 1. Baseline Overlap Frequency

Over 90 consecutive days, 739 MD rounding events were recorded. To analyze the frequency of MD rounds that overlapped with a bedside nurse, only the single-bed patient rooms were examined to reduce false positive overlap. Of the 267 events that took place in single-bed rooms, 80 involved a bedside nurse, for a MD-RN overlap rate of $30.0 \%$. Lengths of time are shown \pm SEM.

rate of overlap is an important first step to establishing bedside interdisciplinary rounding.

\section{METHODS}

\section{Setting and Participants}

The investigation was conducted at a single quaternary-care academic center. The study is exempt per our Institutional Review Board. Data were gathered from three adjacent medical-surgical acute care wards. The layout for each ward was the same: 19 single- or double-occupancy patient rooms arranged in a linear hallway, with a nursing station located at the center of the ward.

The study utilized wearable RFID tags (manufactured by Hill-Rom Holdings, Inc) that located specific staff within the hospital in real time. The RFID tags were checked at Hill-Rom graphical stations to ensure that their locations were tracked accurately. The investigators also wore them and walked around the wards in a prescripted manner to ensure validity. In addition, the locator accuracy was audited by participating attendings once per week and cross-checked with the generated data. Attending physicians on the University Hospitalist inpatient medicine teams were then given their uniquely-tagged RFIDs at the beginning of this study. Nurses already wear individual RFID tags as part of their normal standard-of-care workflow.

The attending hospitalists wore their RFID tags when they were on service for the entirety of the shift. They were encouraged to include nurses at the bedside, but this was not mandatory. The rounding team also included residents and medical students. Rounding usually begins at a prespecified time, but the route taken varies daily depending on patient location. Afternoon rounds were done as needed, depending on patient acuity. The attending physicians' participation in this study was not disclosed to the patient. The patient care activities and daily routines of both nurses and physicians were otherwise unaltered.

\section{Study Design and Data Collection}

Data were collected on the three wards for 90 consecutive days, including nights and weekends. As physicians and nurses moved throughout the ward to conduct their usual patient care activities, the temporal-spatial data associated with their unique RFIDs were automatically collected in real time by the Hill-Rom receivers built into each patient room. Every day, a spreadsheet detailing the activity of all participating nurses and physicians for the past 24 hours was generated for the investigators.

A rounding event was defined as any episode in which a physician was in a patient room for more than 10 seconds. Incidences in which a physician entered and left a room multiple times over a short time span (with less than five minutes in between each event) were classified as a single rounding event. A physician and a nurse were defined as having overlapped if their RFID data showed that they were in the same patient room for a minimum of 10 seconds at the same time. For the purposes of this study, data generated from other RFID-wearing professionals, such as nursing assistants or unit secretaries, as well as data collected from the hallways, were excluded.

\section{Statistical Analysis}

All statistical analyses were conducted using GraphPad Prism (GraphPad Software, San Diego, California). Rounding and overlap lengths were rounded to the nearest minute (minimum one minute). Mean lengths are expressed along with the standard error. Comparisons of the average lengths of MD rounding events between wards was conducted using twotailed Student t-test or one-way ANOVA. Comparisons of the frequency of MD-RN overlap between wards and across different days of the week were performed using a Chi-squared test. The analysis of correlation between the frequency of MD-RN overlap and distance between patient room and nursing station was conducted by calculating Pearson's correlation. A P value of less than .05 was considered statistically significant. 

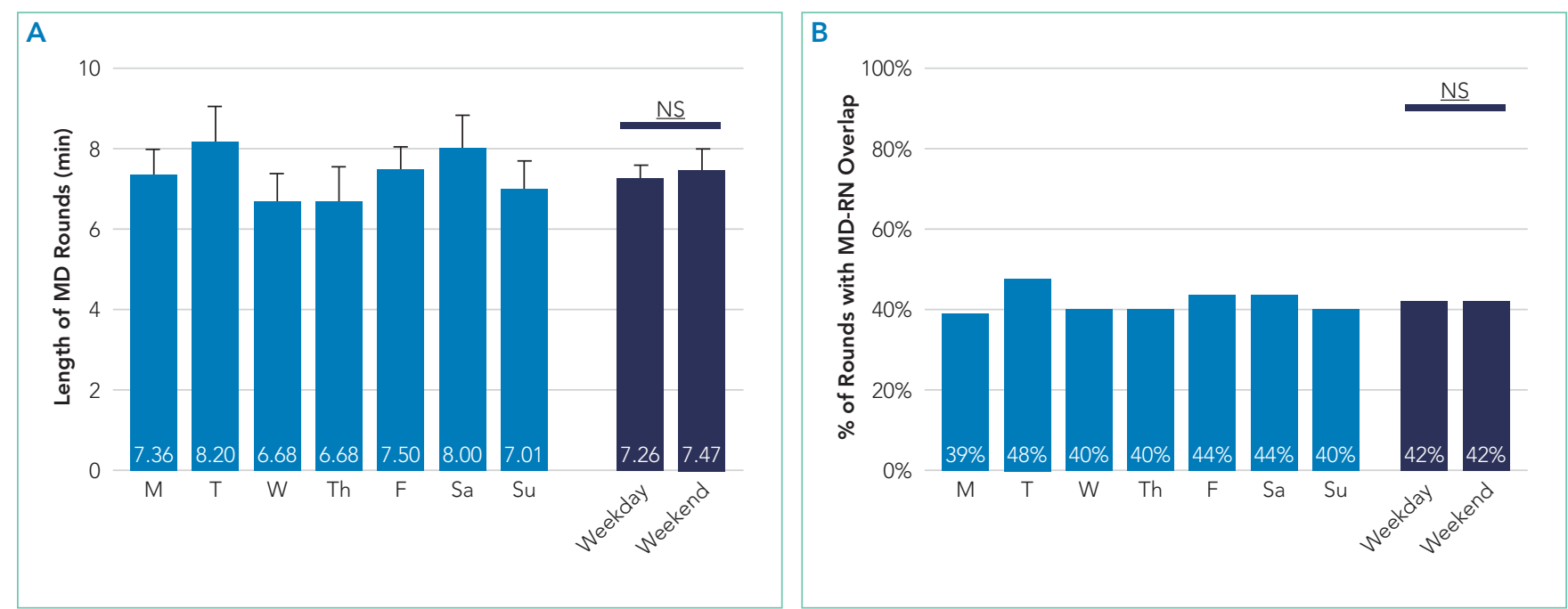

FIG 2. Rounding Characteristics by Day of Week

Data on the length of MD rounds and the frequency of MD-RN overlap for all rooms in the study were partitioned according to day of week. Monday through Friday data were then averaged as "Weekday" and Saturday and Sunday data were averaged as "Weekend." (A) Length of MD rounds by day of week. Mean rounding length in minutes are shown at the base of the bar graphs. Error bars represent standard error. (B) Frequency of MD-RN overlap by day of week. Frequencies of overlap are shown at the base of the bar graphs.

Abbreviation: NS; not statistically significant.

\section{RESULTS}

\section{Baseline Rounding Characteristics}

Over the study period of 90 consecutive days, 739 MD rounding events were captured, for an average of 8.2 events per day. The mean length of all MD rounding events was 7.31 minutes ( \pm 0.27 , ranging from one to 70 minutes). Of these 739 MD rounding events, we separately examined the 267 events that took place in single-bed patient rooms, to control for false-positive physician and nurse interactions (for example, if the MD and RN were caring for two separate roommates). The average rounding length of single-bed rooms was $6.93( \pm 0.27)$ minutes (Figure 1). For the three individual wards, the average rounding lengths were $6.40 \pm 0.73,7.48 \pm 0.94$, and $7.02 \pm 0.54$ minutes, respectively (no statistically significant difference).

\section{Frequency of MD-RN Overlap}

Of the $267 \mathrm{MD}$ rounding events observed in single-bed rooms, a nurse was present in the room for 80 events (30.0\%). The frequencies of MD-RN overlap in patient rooms were $37.0 \%$ (30/81), 28.0\% (14/50), and 26.5\% (36/136) for the three individual wards $(P>.05)$, respectively.

The durations of MD-RN overlap, when these events did occur, were $3.43 \pm 0.38,3.00 \pm 0.70$, and $3.69 \pm 0.92$ minutes, respectively $(P>$.05). The overall mean length of MD-RN overlap for all single rooms was $3.48 \pm 0.45$ minutes.

\section{Rounding Characteristics over the Course of the Week}

To assess how rounding characteristics differed over the work week, we partitioned our data into the individual days of the week. The length of each MD rounding event (time spent in each patient room) did not vary significantly over the course of the week (Figure 2a). When the data for the individual days were aggregated into "weekdays" (Monday through Friday) and "weekends" (Saturday and Sunday), the mean lengths of MD rounds were $7.26 \pm 0.32$ minutes on weekdays and $7.47 \pm$ 0.52 minutes on weekends $(P>.05)$.

In addition, there was no difference in how frequently physicians and nurses overlapped at the patient bedside between weekdays and weekends. Of the 565 weekday MD rounding events, 238 had a nurse at bedside (42.1\%), and of the 173 weekend MD rounding events, 73 had a nurse at bedside (42.2\%; Figure 2b).

\section{Effect of a Bedside Nurse on the Length of Rounds}

Next, the data on the length of MD rounds were partitioned based on whether there was a bedside nurse present during rounds. The mean length of rounds with only MDs (without a bedside nurse) was $5.68 \pm 0.24$ minutes. By comparison, the mean length of rounds with both a nurse and a physician at the patient bedside was $9.56 \pm 0.53$ minutes (Figure 3 ). This difference was statistically significant $(P<.001)$.

\section{Association between Patient Room Location and the Likelihood of MD-RN Overlap}

All three wards in this study have a linear layout, consisting of 19 patient rooms in a row (Figure 4a). The nursing station is located in a central position within each ward, across from the $10^{\text {th }}$ patient room. The frequency of MD-RN overlap was calculated for each room, and each room was ranked according to its relative distance from the nursing station. For each individual ward, there was no statistically significant trend in MD-RN overlap frequency as a function of the distance to the nursing station (data not shown). However, when the data from all three wards were aggregated, there was a statistically significant trend $(P<.05)$ with a negative Pearson correlation 


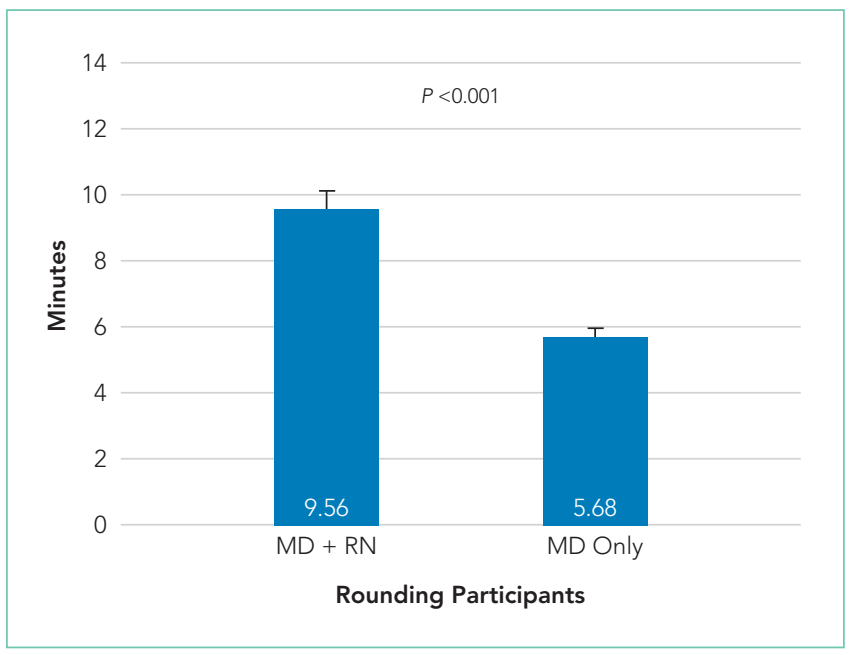

FIG 3. Effect of Bedside Nurse on Rounding Length

All rounding events were characterized into two groups: those with only the hospitalist attending's team (MD Only), and those that included a bedside nurse $(M D+R N)$. The mean rounding length in minutes for each group are shown at the base of the bar graphs. Error bars represent standard error. A twotailed student t-test was used to compare the mean rounding lengths.

$(r=-0.670$; Figure 4b). The slope of the best fit line was 1.94, suggesting that for each additional room farther away from the nursing station, the likelihood of interdisciplinary rounds (with both physicians and nurses together at the bedside) decreases by almost $2 \%$.

\section{DISCUSSION}

To the best of our knowledge, this is the first time-motion study of MD-RN overlap using real-time, RFID-based location technology to capture the rounding activity of both nurses and physicians. Our primary interest was to examine the extent of MD-RN overlap at the patient bedside. This is an important metric that can pave the way for bedside interdisciplinary rounds. Although the exact nature of nurse-physician communication was not measured using the methodology in this study, understanding the length of time physicians spend in patient rooms, across different wards and throughout the work week, provides insights on the current workflow and potential areas of improvement. For example, we found that $30.0 \%$ of MD rounds overlapped with a nurse at the bedside. This baseline data highlight one potential barrier to institution-wide bedside interdisciplinary rounds. Workflow changes, such as better co-localization of patients by service lines or utilization of technologies to augment the visibility of rounding physicians, may improve this overlap frequency.

Data in the literature regarding how much interaction physicians and nurses have, especially at the bedside, are sparse and vary widely. In a recent study using medical students as observers by Stickrath et al., 807 MD rounding events led by medicine attendings were observed over 90 days. The frequency of rounding events that included "communication with nurse" was only $12 \% .{ }^{19}$ Furthermore, only $64.9 \%$ of these communications were at the bedside, for an effective prevalence of bedside MD-RN communication of $7.8 \%$. This number is low compared to our observed frequency of $30.0 \%$. On the other extreme, a study from a hospital that intentionally institutes multidisciplinary rounding (explicitly defined as involving a physician and a nurse at a bedside) reported a frequency range of $63 \%$ to $81 \%$. $^{7}$ A follow-up study by the same group again demonstrated a high frequency of multidisciplinary rounds (74\%) across a variety of ward and specialty types (range $35 \%$ to $97 \%$.). ${ }^{11}$ However, because of the selection bias of this particular setting, the high prevalence does not reflect a generalizable frequency of bedside MD-RN overlap at most hospitals.

The length of time spent by physicians at the patient bedside balances the competing demands of patient care and rapport-building with maintaining efficiency and progressing to other important tasks. In our study, physicians spent an av-

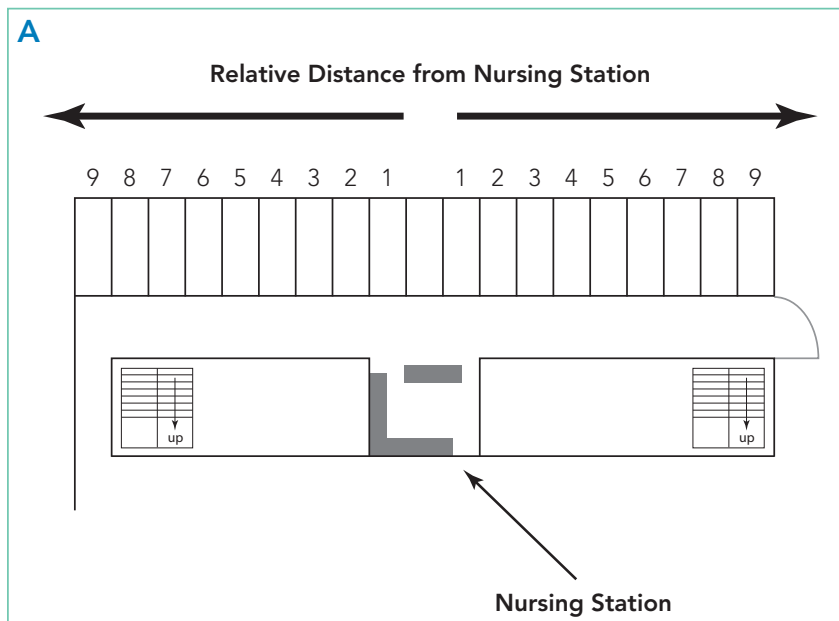

B

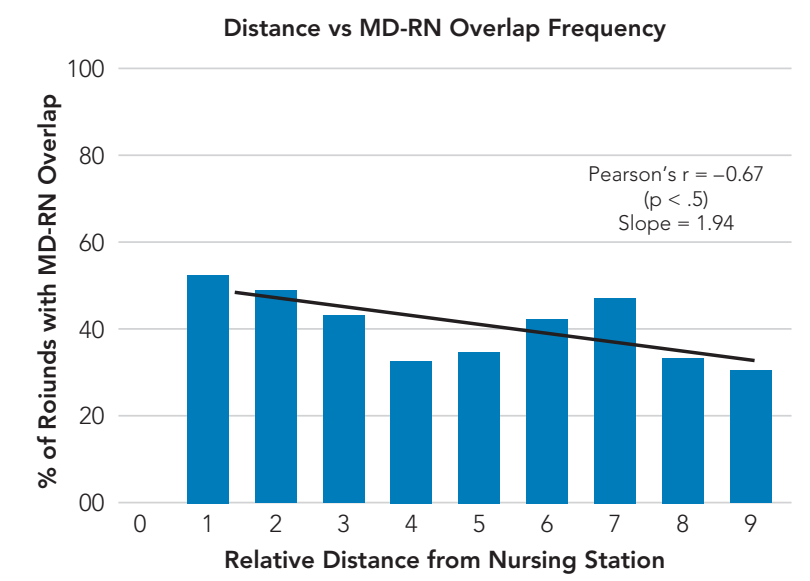

FIG 4. Room Location Affects Likelihood of Interdisciplinary RoundsData on the Frequency of MD-RN Overlap at the Bedside.

Data were analyzed for each patient room, and each patient room was then ranked according to its relative distance from the central nursing station. (A) Schematic layout of the inpatient ward. 19 patient rooms are arranged in a linear hallway, with a nursing station in the center of the ward. (B) Frequency of MD-RN overlap is shown for each group of rooms arranged by their distance from the nursing station. The solid line represents the best-fit line (slope), with a value of -1.94 . 
erage of 7.31 minutes at the bedside per patient. A previously published multiinstitutional observational study, which included our hospital, reported that the average length of rounds at bedside was 4.8 minutes. ${ }^{13}$ A second study reported that 8.0 minutes were spent at the bedside per patient. ${ }^{7}$ All three studies examined the same setting of internal medicine rounds at academic university-based hospitals, led by an attending physician with junior and senior residents present. However, the methodologies to measure the length of physician rounds were different: Priest et al. involved observers, Gonzalos et al. used E-mail-based surveys, and we utilized RFID-based locators. Additional institutional, individual, and patient-based factors also influence the length of rounds and are challenging to directly measure.

Furthermore, the discovery that the length of rounds and the frequency of MD-RN overlap did not statistically differ between weekdays and weekends $(P>$.05) was unexpected. Given the general trend of reduced physician staffing on weekends and the practice of cross-covering larger patient censuses, we would have expected shorter rounds and less frequent MD-RN overlap on the weekends., ${ }^{7,20}$ The remarkable similarity between weekday and weekend metrics suggests that our workflow and rounding habits are not compromised on the weekends.

In addition, we found that MD rounds with a nurse at bedside took longer than rounds without a nurse, and that patient rooms located farther away from the central nursing station had a lower frequency of MD-RN overlap. However, we want to emphasize that these findings are merely associative, and not causal. For example, sicker patients usually take longer to round on than stable patients, and it is also the sicker patients who are more likely to have their nurses at the bedside, independent of physician rounding activity. Furthermore, even if rounding with nurses takes more time, it may ultimately result in fewer pages and overall time savings for both physicians and nurses. 6

With regards to the association between room location and frequency of MD-RN overlap, the data can be interpreted in two ways. On the one hand, if the distance between the patient room and the nursing station does, in fact, reduce the frequency of overlap by almost $2 \%$ per room (Figure $4 b$ ), these data can be informative for future workflow development, quality improvement projects, or even hospital design. On the other hand, many wards might intentionally place more stable, less acute patients farther away from the nursing station because they do not need to be watched as closely. In that case, these data confirm their expectations and no action is needed.

There are several limitations to our study. The principal limitation, as discussed above, is that while our RFID system can generate large quantities of precise data on MD-RN overlap, we do not know the qualitative nature of the overlap. Just because a nurse and a physician are in the same room at the same time does not mean that they are communicating with each other. Second, we defined "rounding" as lasting a minimum of 10 seconds at the bedside. We believe that at least 10 seconds is needed to engage in any meaningful interaction between the physician and the patient, or the physician and the nurse. Reducing the time cutoff below 10 seconds risks capturing more "noise," (decreasing specificity) whereas increasing the time cutoff above 10 seconds risks losing out on encounters that actually had substantial communication (decreasing sensitivity). Even if the communications can be classified as pure "social check-ins," we believe these are important data to capture, as social check-ins are an important part of the patient's care and experience. Third, several studies have commented on the modest accuracy of RFID technology as a locator system. ${ }^{15,21}$ To address this, we both validated the accuracy of our RFID tags prior to the study and restricted our measurements to only inside patient rooms, which has less signal noise than hallways.

Future directions include expanding this study to include housestaff and physicians from other specialities, which may reveal different patterns and metrics of patient and nurse interactions.

\section{CONCLUSION}

RFID technology is a high-throughput method of generating precise, quantitative, and objective data on physician and nurse rounding habits. This tool can be widely applied to generate baseline rounding and overlap data for a variety of wards and settings, especially for institutions that are interested in comparing their metrics and performance to other peer wards or hospitals. Furthermore, this method can generate the necessary pre- and postintervention data for countless quality improvement endeavors, including efforts to enhance bedside interdisciplinary rounding.

\section{Acknowledgments}

The authors would like to thank the attending hospitalists who piloted wearing the RFID tags. This study would not be possible without your participation. The authors also wish to extend their appreciation to Gretchen Brown, MSN RN NEA-BC, for her support. Finally, the authors would like to thank Dr. Laurence Katznelson, Thi Dinh La, and the Resident Safety Council at Stanford, as well as the Stanford GME Office.

Disclosures: The authors have nothing to disclose.

\section{References}

1. Knaus WA, Draper EA, Wagner DP, Zimmerman JE. An evaluation of outcome from intensive care in major medical centers. Ann Intern Med. 1986;104(3):410-418. https://doi.org/10.7326/0003-4819-104-3-410.

2. Larrabee JH, Ostrow CL, Withrow ML, Janney MA, Hobbs GR, Burant C. Predictors of patient satisfaction with inpatient hospital nursing care. Res Nurs Health. 2004;27(4):254-268. https://doi.org/10.1002/nur.20021.

3. Rosenstein $\mathrm{AH}$. Nurse-physician relationships: impact on nurse satisfaction and retention. AJN Am J Nurs. 2002;102(6):26-34.

4. Galletta M, Portoghese I, Battistelli A, Leiter MP. The roles of unit leadership and nurse-physician collaboration on nursing turnover intention. J Adv Nurs. 2013;69(8):1771-1784. https://doi.org/10.1111/jan.12039.

5. Wanzer MB, Wojtaszczyk AM, Kelly J. Nurses' perceptions of physicians' communication: the relationship among communication practices, satisfaction, and collaboration. Health Commun. 2009;24(8):683-691. https://doi. org/10.1080/10410230903263990.

6. Ratelle J, Henkin S, Chon T, Christopherson M, Halvorsen A, Worden L. Improving nurse-physician teamwork through interprofessional bedside rounding. J Multidiscip Healthc. 2016;9:201. https://doi.org/10.2147/JMDH.S106644.

7. Gonzalo JD, Kuperman E, Lehman E, Haidet P. Bedside interprofession- 
al rounds: perceptions of benefits and barriers by internal medicine nursing staff, attending physicians, and housestaff physicians. J Hosp Med. 2014;9(10):646-651. https://doi.org/10.1002/jhm.2245.

8. Rimmerman $\mathrm{CM}$. Establishing patient-centered physician and nurse bedside rounding. Physician Exec. 2013;39(3):22.

9. Curley $C$, McEachern JE, Speroff T. A firm trial of interdisciplinary rounds on the inpatient medical wards: an intervention designed using continuous quality improvement. Med Care. 1998;36(8):AS4-AS12.

10. Rothberg MB, Steele JR, Wheeler J, Arora A, Priya A, Lindenauer PK. The relationship between time spent communicating and communication outcomes on a hospital medicine service. J Gen Intern Med. 2012;27(2):185-189. https://doi.org/10.1007/s11606-011-1857-8.

11. Gonzalo JD, Himes J, McGillen B, Shifflet V, Lehman E. Interprofessional collaborative care characteristics and the occurrence of bedside interprofessional rounds: a cross-sectional analysis. BMC Health Serv Res. 2016;16(1):459. https://doi.org/10.1186/s12913-016-1714-x.

12. Nair DM, Fitzpatrick JJ, McNulty R, Click ER, Glembocki MM. Frequency of nurse-physician collaborative behaviors in an acute care hospital. J Interprof Care. 2012;26(2):115-120. https://doi.org/10.3109/13561820.2011.637647.

13. Priest JR, Bereknyei S, Hooper K, Braddock CH. Relationships of the location and content of rounds to specialty, institution, patient-census, and team Size. PLoS One. 2010;5(6):e11246. https://doi.org/10.1371/journal.pone.0011246.

14. Li L, Hains I, Hordern T, Milliss D, Raper R, Westbrook J. What do ICU doctors do?: a multisite time and motion study of the clinical work patterns of regis- trars. Crit Care Resusc. 2015;17(3):159.

15. Okoniewska B, Graham A, Gavrilova M, et al. Multidimensional evaluation of a radio frequency identification wi-fi location tracking system in an acutecare hospital setting. J Am Med Inform Assoc. 2012;19(4):674-679. https:// doi.org/10.1136/amiajnl-2011-000560.

16. Ward DR, Ghali WA, Graham A, Lemaire JB. A real-time locating system observes physician time-motion patterns during walk-rounds: a pilot study. BMC Med Educ. 2014;14:37. https://doi.org/10.1186/1472-6920-14-37.

17. Fahey L, Dunn Lopez K, Storfjell J, Keenan G. Expanding potential of radiofrequency nurse call systems to measure nursing time in patient rooms. J Nurs Adm. 2013;43(5):302-307. https://doi.org/10.1097/NNA.0b013e31828eebe1.

18. Hendrich A, Chow M, Skierczynski BA, Lu Z. A 36-hospital time and motion study: how do medical-surgical nurses spend their time? Perm J. 2008:50.

19. Stickrath C, Noble M, Prochazka A, et al. Attending rounds in the current era: what is and is not happening. JAMA Intern Med. 2013;173(12):1084. https:// doi.org/10.1001/jamainternmed.2013.6041.

20. Blecker S, Goldfeld K, Park H, et al. Impact of an intervention to improve weekend hospital care at an academic medical center: an observational study. J Gen Intern Med. 2015;30(11):1657-1664. https://doi.org/10.1007/ s11606-015-3330-6

21. Westbrook JI, Duffield C, Li L, Creswick NJ. How much time do nurses have for patients? A longitudinal study quantifying hospital nurses' patterns of task time distribution and interactions with health professionals. BMC Health Serv Res. 2011;11(1):319. https://doi.org/10.1186/1472-6963-11-319. 